\title{
RETRACTED CHAPTER: A Survey \\ on Detection and Mitigation \\ of Distributed Denial-of-Service Attack \\ in Named Data Networking
}

\section{Sandesh Rai, Kalpana Sharma and Dependra Dhakal}

The editors have retracted this article [1] because Figures 1 and as well as parts of the text, were duplicated from a previously published arti n by the same authors [2]. In addition, Figure 3 was duplicated from a pre ious $1 / y$ published article by Afanasyev et al [3]. This article is therefore redun $\mathrm{nt}$.

All authors agree to this retraction.

1. [Reference to https://link.springer.com/chap /10.1007/978-981-10-8911-4_18]

2. [Reference to https://link.springer.copa// apter/10.1007/978-981-10-8237-5_51]

3. Afanasyev, P. Mahadevan, I. Mo1s nk, E. Uzun and L. Zhang, "Interest flooding attack and counterme ures it Named Data Networking," 2013 IFIP Networking Conference, Brøokly D.Y, 2013, pp. 1-9. 